Gut, 1972, 13, 427-429

\title{
Cell-mediated immune reactivity in liver disease
}

\author{
P. A. BACON, H. BERRY, AND R. BOWN \\ From the Departments of Experimental Pathology and Gastroenterology, St Bartholomew's Hospital, London
}

SUMMARY Cell-mediated immunity has been investigated using the leucocyte migration test in 32 patients with chronic active hepatitis or primary biliary cirrhosis. Twenty-six (75\%) showed reactivity to liver extract. At least two antigens are involved. Cell-mediated reactivity was confirmed in some cases using lymphocyte 'blast' transformation. There was no correlation with the presence of autoantibodies.

Cell-mediated immunity to parotid extract was shown in 10 cases $(28 \%)$. This is in accord with the occurrence of the sicca syndrome in liver disease, which has been confirmed in this study.

There is extensive evidence of altered immune reactivity in some types of liver disease. This has been recently reviewed by Doniach (1970), who put forward a unified concept of autoimmune liver disease. Mitochondrial antibody, smooth muscle antibody, and antinuclear factors (ANF) have been demonstrated in primary biliary cirrhosis and chronic active hepatitis but the pathogenic significance of these autoantibodies is uncertain. The histology of these diseases shows infiltration with small lymphocytes but specific cell-mediated immune reactivity has been little studied so far. We have studied cell-mediated immune reactivity in vitro in various liver diseases using liver and parotid extracts as antigens. The latter was chosen in view of the known association of the sicca syndrome with liver disease (Golding, Bown, Mason, and Taylor, 1970).

\section{Method}

Patients attending the gastroenterology departments of St Bartholomew's Hospital or of Sheffield Royal Infirmary were studied. Eighteen patients with primary biliary cirrhosis and 14 patients with chronic active hepatitis ('autoimmune liver group') were tested, together with 19 patients with other liver disease (alcoholic cirrhosis, obstructive jaundice etc), 20 control patients with other disease, and 10 healthy adults. The diagnosis in the first groups was confirmed by liver biopsy in all cases.

Cell-mediated immune reactivity was assessed in vitro using the leucocyte migration test of Soberg and Bendixen (1969) in which separated peripheral blood leucocytes packed into capillary tubes are allowed to migrate out into $1 \mathrm{ml}$ culture chambers Received for publication 28 March 1972. containing antigen in culture medium. Results are expressed as the percentage migration in test chambers containing antigen compared to control chambers without antigen. Both stimulation and inhibition of migration indicate biological reactivity in this test (Søborg and Bendixen, 1967). A result of greater than $120 \%$ or less than $80 \%$ is taken as significant. In order to give a uniform scale all results over $100 \%$ have been inverted so that $120 \%$ and $80 \%$ both appear as a migration index of 80 .

Previous work has shown lymphocyte blast transformation to autologous liver tissue (Tobias, Safran, and Schaffner, 1967) and also to ribosomal fraction (Pipitone, 1967). We sought to compare leucocyte migration test results with in-vitro lymphocyte transformation using both foetal mitochondrial extract and the liver extract in a proportion of cases. Reactivity was measured as the uptake of $\mathrm{H}_{3}$ thymidine using liquid scintillation counting.

The antigens used were derived from fresh cadaver liver and parotid gland from a patient dying of trauma. They were extracted by homogenization and sonication. The extracts were stored after freeze drying and reconstituted before use. Two hundred $\mu \mathrm{g}$ of protein extract was used routinely, as this had been shown in initial dilution experiments not to affect cells from healthy controls. A liver mitochondrial preparation extracted from fresh foetal tissue was also used in some cases.

Mitochondrial antibody, smooth muscle antibody, and antinuclear factors were examined for by immunofluorescence.

The assessment of the sicca syndrome was made by measuring salivary secretion as the five-minute collection during stimulation with chewing gum and lachrymal secretion by Shirmer filter paper strips 
without other stimulation. Conjunctival staining with Rose-Bengal was also sought.

\section{Results}

Reactivity to liver antigen in the leucocyte migration tests (Fig. 1) was shown by $14(78 \%)$ patients with primary biliary cirrhosis and $12(75 \%)$ patients with chronic active hepatitis but only one patient with other liver disease and none of the controls. The migration index showed no correlation with biochemical indices of liver disease (such as bilirubin, transaminases, or alkaline phosphatase) with age of the patient or with duration of disease. The four non-reacting patients with primary biliary cirrhosis had fairly early disease (under four years) but other patients showed strong reactivity at this stage. There was also no correlation with the presence of autoantibodies so that a similar proportion of reactors in the leucocyte migration test was seen in those with and without each of the three autoantibodies.

Eighteen patients with 'autoimmune liver disease' were tested with both foetal liver mitochondrial preparation and liver extract using the leucocyte migration test and 11 patients showed reactivity to both liver and mitochondrial extract. One patient showed reactivity to both liver and mitochondrial extract. One patient showed reactivity to the mitochondrial extract but no response to liver extract. Three patients showed response to liver extract but no response to mitochondrialextractand two patients showed no response to either extract (Fig. 2).

Lymphocyte transformation tests were performed

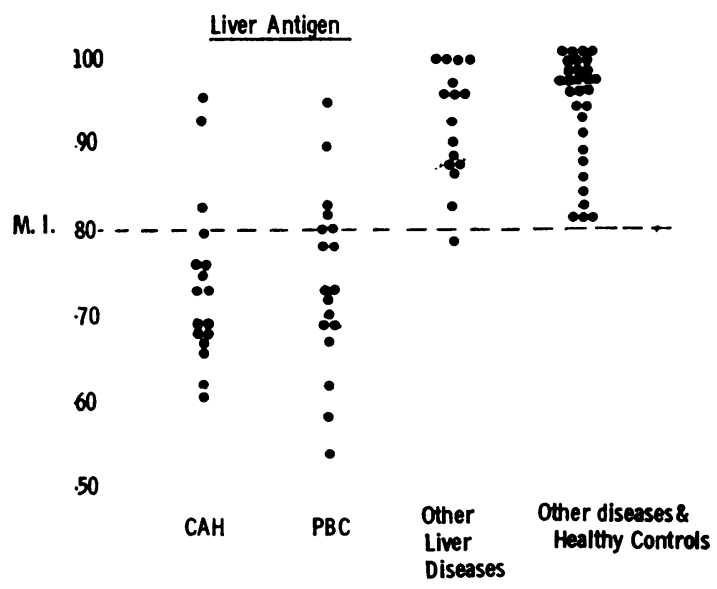

Fig. 1 The migration index (M.I.) to liver antigen in 'autoimmune' and other liver diseases.

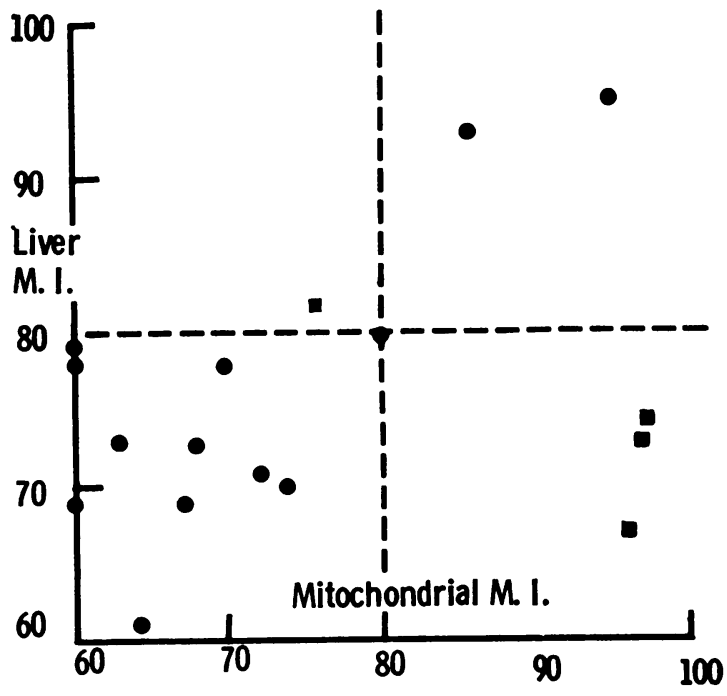

Fig. 2 A comparison of the migration index using the liver antigens in 17 patients. Only four $(\square)$ did not give the same result with both antigens. The rest $(O)$ were in accord.

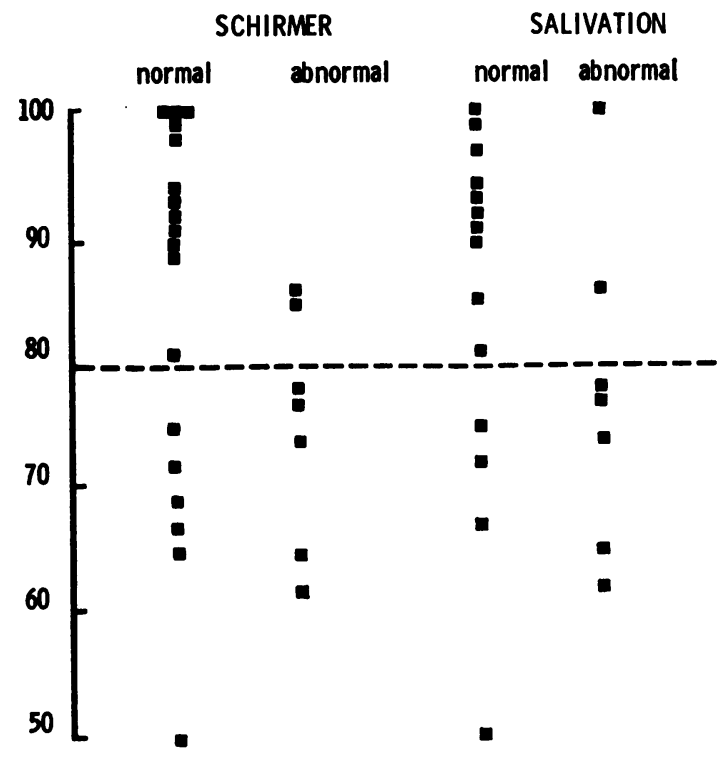

Fig. 3 The relationship between clinical aspects of the sicca syndrome and the migration index using parotid antigen. Ten patients showed depression of one or both aspects. 
in nine patients with 'autoimmune liver disease' all of whom showed a positive leucocyte migration test to liver and mitochondrial extract. Five reacted to the whole liver extract and four to the mitochondrial preparation. Two of the nine patients showed transformation to both extracts, three showed transformation to only liver extract, and a further two showed transformation to mitochondrial extract only. None of the four controls reacted to either antigen.

Reactivity to parotid antigen was seen in six $(33 \%)$ patients with primary biliary cirrhosis and four $(27 \%)$ patients with chronic active hepatitis. One patient with other liver disease reacted but none of the controls. Clinical evidence of some aspect of the sicca syndrome was found in $10(43 \%)$ patients with 'autoimmune disease' out of the 24 thus tested (Fig. 3). Five (50\%) of these reacted to parotid but so did four $(35 \%)$ of the patients with no clinical evidence of sicca. One of these latter patients showed diminished lachrymal secretion on retesting six months later.

\section{Discussion}

This study has shown that $75 \%$ of patients with an 'autoimmune' type of liver disease have evidence in vitro of cell-mediated immunity directed against liver antigens. This reactivity is directed against foetal as well as adult tissue and at least two antigens are involved. The lack of correlation with autoantibodies suggests that this reactivity is not simply another marker of tissue damage. However, the lack of correlation with disease duration or state makes assessment of the pathogenic significance difficult. It is probable that further antigenic purification would help to clarify this point. These findings lend support to the suggestion (Popper, Paronetto, and Schaffer, 1965) that primary biliary cirrhosis may represent a chronic delayed hypersensitivity reaction directed against liver or biliary tissue.

Evidence of the sicca syndrome, as shown by diminished salivary or lachrymal secretion, was seen less often in this group than in the study of Golding et al (1970). This may represent differences in patient selection or stage of disease as several fatal cases in this series had not been tested. The incidence of cellular hypersensitivity to parotid in the group of patients with clinical sicca components was very close to that described in patients with classical Söjgren's disease (Søborg and Bertram, 1968) but the numbers are small. The incidence of reactivity in patients without clinical sicca involvement is of interest. It was virtually confined to the 'autoimmune' liver group and may represent a preclinical stage of sicca involvement. This is supported by the patient who progressed to clinical involvement under observation.

The relationship of liver disease to the sicca syndrome may represent a common autoimmune pathogenic mechanism. An overlap of rheumatoid arthritis (Whaley, Williamson, Dick, Gondie, Nuki, and Buchanan, 1970) and scleroderma (Murray Lyon, Thomson, Ansell, and Williams, 1970) with primary biliary cirrhosis has been described and both these diseases are more commonly accepted components of Sjögren's syndrome. Alternatively both liver and sicca involvement could be secondary to a prior alteration of immune reactivity, as suggested by the depression of contact hypersensitivity in D.N.C.B. seen in both Sjögren's disease (Leventhal, Waldorf, and Talal, 1967) and primary biliary cirrhosis (Fox, Schever, James, Scharma, and Sherlock, 1969).

Our thanks are due to Dr A. M. Dawson and Dr D. Holdsworth (Sheffield Royal Infirmary) for permission to study patients under their care. We are grateful to Dr Enid Taylor for the conjunctival staining studies. H.B. is in receipt of an Aylwen bursary from St Bartholomew's Hospital, and we are grateful to the Governors of the Hospital for their support.

\section{References}

Doniach, D. (1970). The concept of an 'autoallergic' hepatitis. Proc. roy. Soc. Med., 63, 527-531.

Fox, R. A., Schever, P. J., James, D. G., Scharma, O., and Sherlock, S. (1969). Impaired delayed hypersensitivity in primary biliary cirrhosis. Lancet, 1, 959-962.

Golding, P. J., Bown, R., Mason, A. M. S., and Taylor, E. (1970). 'Sicca complex' in liver disease. Brit. med. J., 4, 340-342.

Leventhal, B. G., Waldorf, D. S., and Talal, N. (1967). Impaired lymphocyte transformation and delayed hypersensitivity in Sjögren's syndrome. J. clin. Invest., 46, 1338-1345.

Murray Lyon, I. M., Thompson, R. P. H., Ansell, I. D., and Williams, R. (1970). Scleroderma and primary biliary cirrhosis. Brit. med. J., 3, 258-259.

Pipitone, V. (1967). Lymphocyte stimulation and liver disease. Lancet, 1, 849-850.

Popper, H., Paronetto, F., and Schaffer, F. (1965). Immune processes in the pathogenisis of liver disease. Ann. N.Y. Acad. Sci., 124, 781-799.

Søborg, M., and Bendixen, G. (1967). Human lymphcocyte migration as a parameter of hypersensitivity. Acta med. Scand., 181, 247256.

Søborg, M., and Bendixen, G. (1969). A leucocyte migration technique for in vitro detection of cellular (delayed type) hypersensitivity in man-a survey. Dan. med. Bull., 16, 1-6.

Søborg, M., and Betram, U. (1968). Cellular hypersensitivity in Sjögren's syndrome. Acta med. scand., 184, 319-322.

Tobias, H., Safran, A. P., and Schaffner, F. (1967). Lymphocyte stimulation and chronic liver disease. Lancet, 1, 193-195.

Whaley, K., Williamson, J., Dick, W. C., Goudie, R. B., Nuki, G. and Buchanan, W. W. (1970). Liver disease in Sjögren's syndrome and rheumatoid arthritis. Lancet, 1, 861-863. 